(Aus der medizinischen Klinik der Universität Heidelberg.)

\title{
Über Wärmetönungen chemischer Prozesse in lebenden Zellen.
}

\author{
(Versuche an Blutzellen.) \\ Von \\ otto Meyerhor.
}

(Hierzu Tafel II.)

Die vorliegende Arbeit schliesst sich in sachlicher und methodischer Hinsicht eng an die Untersuchungen über die Wärmetönung der vitalen Oxydationsvorgänge am Ei und Samen des Seeigels und den Eiern und Larven der Aplysia an ${ }^{1}$ ). Die Benutzung von Vogel-Erythrocyten, die einen leicht messbaren Stoffwechsel haben, ergab sich aus verschiedenen Gesichtspunkten. Einmal waren die Beeinflussungen der Oxydationsgeschwindigkeit hier von o. Warburg ebenso weitgehend studiert worden wie am Seeigelei ${ }^{2}$ ), und es liessen sich einige in obengenannter Arbeit nicht oder ungenügend berücksichtigte Punkte dabei aufklären; ferner handelt es sich hier um einzellige keinerlei sichtbare Veränderungen eingehende, ihrem Wesen nach innerhalb gewisser Zeiten stabil erscheinende Organismen, bei denen unter normalen Verhältnissen als kalorischer Quotient ${ }^{3}$ ) (Anzahl von Grammkalorien pro Verbrauch von $1 \mathrm{mg} \mathrm{O}_{2}$ ) der Verbrennungswert von Eiweiss, Fett oder Kohlehydraten zu erwarten war. Es wurde ferner vorausgesetzt, dass bei dem grossen Sauerstoffvorrat im Hämoglobin die Ausschläge der einzelnen Messungen

1) Otto Meyerhof, Untersuchungen über die Wärmetönung der vitalen Oxydationsvorgänge in Eiern. I.-III. Biochem. Zeitschr. Bd. 35 S. 246. 1911.

2) 0. Warburg, Zeitschr. f. physiol. Chemie Bd. 59 S. 112, Bd. 69 S. .452, Bd. 70 S. 413 , Bd. 76 S. 331 . Verhandlungen des deutschen Kongresses für innere Medizin Bd. 28 S. 553. 1911. Münchener med. Wochenschr. 1911 Nr. 6. Warburg und Wiesel, Pflüger's Arch. Bd. 144 S. 465. 1912. - Onaka, Zeitschr. f. physiol. Chemie Bd. 70 S. 433.

3) Siehe Biochem. Zeitschr. Bd. 35 S. 253.

Pflügør's Archiv für Plysiologie. Bd. 146. 
genügend gross sein würden, um die Genauigkeit der erhaltenen Konstante weit über die der früheren zu bringen, bei denen der geringe Sauerstoffvorrat der Versuchsflaschen vor allem an der verhältnismässig grossen Fehlerbreite von $\pm 6 \%$ schuld erschien. Jedoch hat sich diese Hoffnung nicht erfült. Erstens erwiesen sich die Wärmemessungen bei höheren Temperaturen als ungenauer wie um Zimmertemperatur; ferner war die Wiukler'sche Sauerstofftitration der hier benutzten Warburg'schen Bestimmung mit dem Barcoft'schen Blutgasmanometer, wenn auch nicht an Empfindlichkeit, so doch, worauf es in unserem Falle allein ankommt, an Fehlergenauigkeit überlegen. Endlich aber entstand durch die Wärmetönung der Hämoglobingasbindungen eine erhebliche Unsicherheit, die nicht ganz vollständig behoben werden konnte. Es muss deshalb als ein nicht zu schlechtes Resultat angesehen werden, dass sämtliche gefundenen Werte auch nicht weiter voneinander abliegen als $15 \%$, so dass hier die Fehlerbreite etwa $\pm 7,5 \%$ beträgt.

Ehe ich die hier gewonnenen Resultate mitteile, muss eine Ungenauigkeit in der Berechnung der erwähnten kalorisehen Grössen in der letzten Untersuchung berichtigt werden, die für das Giesamtresultat in Betracht kommt, wenn sie auch unter die Genauigkeit der einzelnen Experimente fällt. Es war nämlich bei der Berechnung des Wasserwerts die spezifische Wärme des Meerwassers statt mit dem spezifischen Gewicht desselben $(=1,025)$ nur mit dem des Wassers multipliziert, und alle erhaltenen Grössen müssen deshalb unter Berücksichtigung des Wasserwertes des Gefässes auf eine Gesamtkapazität umgerechnet werden, die 2,2\% grösser ist als die angenommene. Für die Versuche in bypertonischen Lösungen, wo dieser Fehler auch gemacht wurde, ist die Korrektur noch etwas grösser. Als Mittel der Versuche am Seeigelei bei normaler Furchung. ergibt sich statt $2,73: 2,79$, reduziert um 0,17 für die Wärmetönung der $\mathrm{CO}_{2}$-Bindung und -Lösung $=2,62$; für Eier mit künstlichen Membranen statt 2,78: 2,84 (-0,17) usw. Bei hypertonischen Lösungen beträgt die Korrektur durchschnittlich $4 \%$, statt im Mittel 2,75 ergibt sich $2,86(-0,17)$. Für Samen von Seeigeln folgt statt 3,24:3,31 (-0,17); für Aplysiaeier statt 2,98:3,05 (-0,17), für Aplysialarven statt 3,08:3,15 (-0,17). Da der kalorische Koeffizient des Sauerstoffs für Eiweissverbrennung 3,2, für Fett 3,3, für Kohlehydrate 3,4-3,5 beträgt, so ist auf diese Weise der Wert für Samen dem Koeffizienten der Eiweissverbrennung annähernd gleich. 


\section{Der kalorische Quotient für die Atmung der Vogelerythrocyten.}

Als Mittel sämtlicher Bestimmungen des kalorischen Quotienten ergibt sich ein Wert zwischen 3,2 und 3,3, und zwar für zehn Wärmemessungen und zwölf parallele Sauerstoffbestimmungen 3,26, für drei Wärmemessungen unter Durchleiten eines Sauerstoffstroms, wodurch die Wärmetönungen der Hämoglobinreduktion und Kohlensäurebindung in Wegfall kommen, und fünf entsprechende Sauerstoffbestimmungen 3,20 , für eine durch Äthylurethau um $35 \%$ gehemmte Atmung bei einer Wärme- und zwei Sauerstoffbestimmungen 3,30. Die Vermutung, dass sich bei der Erythrocytenatmung ein "normaler" Quotient ergeben würde, hat sich also bestätigt. Er liegt zwischen Eiweiss- und Fettverbrennung.

Das Messungsverfahren war im wesentlichen das gleiche wie bei der letzten Untersuchung 1). Im einzelnen waren Modifikationen erforderlich. Der Thermostat war auf $29^{\circ} \mathrm{C}$. reguliert. Während einer mehrstündigen Versuchszeit bestand meist eine Konstanz von $0,02-0,03^{\circ}$ C. Für die Mehrzahl der Wärmeversuche diente ein Dewargefäss (Protokollnummer D IV) von $190 \mathrm{ccm}$ Rauminhalt, das einen Abkühlungskoeffizienten gegen Wasser von 5\% pro 1 Stunde hatte. Für einige Messungen diente das früher benutzte Gefäss (D II) von $240 \mathrm{ccm}$ Inhalt, dessen Abkühlungskoeffizient pro 1 Stunde $3 \%$ betrug; doch standen dafür meist nicht genügende Blutmengen zur Verfügung. Da bei hoher Thermostatentemperatur sich eine Auskühlung gegen das Zimmer längs des Thermometerrohrs geltend machte, so wurde an Stelle des auf dem Dewargefäss befestigten Glaszylinders eine sich nach oben verjüngende, oben offene Glasglocke benutzt, über die ein langer, um das Thermometer festgebundener Gummifinger gezogen war, so dass das Kalorimeter sehr tief, bis zum oberen Rand des Gummifingers in den Thermostaten versenkt werden konnte. Trotzdem und trotz einer längeren Vorwärmung der in der Glocke steckenden Watte war auch dann noch für die ersten 2 Stunden eine geringe Differenz des Abkühlungskoeffizienten für über und unter dem Thermostaten gelegene Temperaturen bemerkbar, und zwar betrug derselbe $4,6-4,8 \%$ für tiefere, $5,3-5,1 \%$ für höhere Temperaturen. Nach 2 Stunden hatte sich dieser Unterschied ausgeglichen. Da es untunlich war, so lange Zeit vor der Messung verstreichen zu lassen, wurden die Anfangszeiten in dieser Richtung korrigiert. Doch musste jedesmal 20-30 Minuten, vom Einhängen in den Thermostaten an gerechnet, gewartet werden, ehe ein regelmässiger Temperaturanstieg erfolgte; bei sehr genauen Messungen, bei denen kleine Ausschläge zu erwarten waren, 40-50 Minuten; aber auch dann war die nächste Stunde aus den erwähnten Gründen noch nicht absolut genau.

In den Versuchen, bei denen Sauerstoff während der Versuchszeit durch das Gefäss geleitet wurde, wurde eine von zwei Glasröhren durchbohrte Glasglocke

1) Vgl. Biochem. Zeitschr. Bd. 35 S. 265. 1911. 
benutzt. An der einen Röhre war aussen das Zuleitungsrohr befestigt, in das der Sauerstoff aus einer Bombe durch mehrere im Thermostaten stehende Waschflaschen und eine lange "Wärmeschlange" gelangte, so dass er genau auf Thermostatentemperatur in die Blutkörperchenlösung kam, in die er durch ein vielfach durchbohrtes Glasrohr strömte. Nach dem Passieren des Versuchsgefässes wurde der Sauerstoff durch das herausführende Rohr unter einen Messzylinder geleitet, so dass seine Menge berechnet werden konnte. Diese wurde so klein gehalten - etwa 1 Liter für 2 Stunden —, dass die für die Wärmekapazität des Sauerstoffs anzubringende Korrektur ausser Betracht kam. - Für die Messungen wurden verschiedene Beckmann-Thermometer benutzt, zumeist ein geprüftes, in $0,01^{\circ} \mathrm{ge}$ teiltes Thermometer, dessen Ablesungen nach dem Prüfungsschein korrigiert wurden; gelegentlich auch ein in $0,002^{\circ}$ geteiltes Thermometer, das aber eine sehr langsame Einstellung zeigte und deshalb unbequem war. Die Ablesung geschah wie früher mit Lupenvergrösserung. Die Sauerstoff bestimmungen wurden nach dem W arburg'schen Verfahren mit dem Haldane-Barcroft'schen Manometer vorgenommen, unter Benutzung der Verbesserungen, die kürzlich beschrieben wurden ${ }^{1}$ ). Es wurden meist Atmungsgefässe von $6,1-6,3 \mathrm{ccm}$, anfangs solche von $3,2 \mathrm{ccm}$ benutzt; gelegentlich kamen auch solche von 10-12 ccm zur Verwendung. Stets wurde vor dem Versuch eine gleiche Menge luftgesättigter Erythrocytensuspension zur Kontrolle mit $0,05 \mathrm{ccm} n$-Cyankali vergiftet; und ebenso nach dem Versuch die Atmungsprobe mit der gleichen Menge Cyankali. Die Grösse der Atmung ergibt sich aus der Differenz der Ausschläge dieser beiden Proben, nach der Formel $v_{0}=\frac{v p}{p_{0}(1+a t)}$ in Kubikzentimeter Sauerstoff unter Normalbedingungen von Temperatur und Atmosphärendruck ( $v$ : Volumen des Absorptionsgefässes plus Capillare minus eingefüllter Flüssigkeit in Kubikzentimeter. $p$ Druckabnahme im Manometer, gemessen in Millimeter Wasser bezw. wässriger Lösung von gallensaurem Natrium (spez. Gew. 1,033), $p_{0}$ Normaldruck in Millimeter der gleichen Lösung $=10000, t=$ Absorptionstemperatur in ${ }^{0} \mathrm{C}$ ). Die Grösse des Ausschlages ist vom Barometerdruck völlig unabhängig und daher direkt ein Maass für die Menge des verschwindenden Sauerstoffs. Diese Menge wird also durch Multiplikation mit 1,43 in Milligramm Sauerstoff erhalten. Da bei grossen Ausschlägen der Sauerstoffgehalt des Absorptionsgefässes erheblich gesunken ist und daher die Sättigang der Flüssigkeit durch den veränderten Partialdruck herabgesetzt ist, wurde für diese Fälle eine entsprechende Korrektur angebracht.

Ehe mit den eigentlichen Messungen begonnen werden konnte oder diese ganz berechenbar waren, mussten eine Reihe von Vormessungen vorgenommen werden. Diese betrafen 1. die Wärmekapazitäten der Anordnung, insbesondere die spezifische Wärme von Vogel-Erythrocyten, 2. die Wärmetönungen der Gasbindungen des

1) Warburg, Zeitschr. f. physiol. Chemie Bd. 76 S. 343 . Siehe auch Bd. 69 S. 457. 
Hämoglobins, 3. für die nötige Korrektur bei dem Ansteigen der Temperatur in dem adiabatischen Kalorimeter die Kenntnis des Temperaturkoeffizienten der Erythrocytenatmung.

Die Ermittlung des Wasserwerts der Gefässe geschah wie früher durch Heizung mit dem elektrischen Strom. Der Strom eines Akkumulators durchfloss - unter Einschaltung regulierbarer Vorschaltwiderstände - einen im Dewargefäss befestigten Manganinwiderstand von 2,92 0hm, während das Gefäss mit einer genau bestimmten Menge Wasser gefüllt war. Spannung und Stromstärke wurden während der Versuchszeit dauernd mit geprüften Weston-Strom- und -Spannungsmessern kontrolliert. Aus dem Temperaturanstieg $(T)$ berechnet sich dann die Wärmekapazität $(C)$ nach der Formel $Q=C \cdot T=0,239 i \cdot e \cdot t$ g-cal. $(i:$ Ampere, $e:$ Volt, $t:$ Sekunden). Da die Gefässe sich während des Versuchs aus äusseren Gründen nicht im Thermostaten, sondern statt dessen nur in einem wattegefüllten Kasten befanden, war die Auskühlung relativ beträchtlich und nicht ganz genau berechenbar, worunter die Genauigkeit der Versuche litt. Bei Füllung des Gefässes D IV mit $180 \mathrm{ccm}$ Wasser ergab sich in zwei Versuchen eine Gesamtkapazität von 198,2 und 200,2, also als Wasserwert des Gefässes 18,2-20,2. Im späteren wird 19,5 dafür genommen. Eine Wiederholung der Eichung des Gefässes D II ergab bei Füllung mit $200 \mathrm{ccm}$ Wasser einen Wasserwert von 22-23, während früher bei Füllung mit $230 \mathrm{ccm}$ Wasser 24 in guter Übereinstimmung dazu gefunden war.

Darauf wurde die spezifische Wärme einer bestimmten Konzentration von Gänse-Erythrocyten in 0,9\%iger NaCl-Lösung ermittelt, aus der für alle späteren Messungen die spezifischen Wärmen der benutzten Konzentrationen interpoliert wurden. Diese Interpolation geschah durch Messung des spezifischen Gewichts der betreffenden Lösungen. Da zu allen Versuchen in 0,9\% iger NaCl-Lösung (spezifisches Gewicht 1006) gewaschene Erythrocyten gebraucht wurden, und man annehmen darf, dass die Erythrocyten im Mittel stets gleich schwer sind, kann das spezifische Gewicht als ein genügend sicherer Index der Menge der Erythrocyten und damit der nur von der Menge der Erythrocyten abhängenden spezifischen Wärme angesehen werden. Die Hämoglobinbestimmungen nach Sahli erwiesen sich für Vergleichszwecke erheblich ungenauer, während die N-Werte dem spezifischen Gewicht einigermaassen parallel zu gehen schieuen ${ }^{1}$ ). Für die Messung der spezifischen Wärme wurde eine dichte Suspension

1) Ausgeführt sind nur Messungen der spezifischen Wärme an Sängetierblut, die genauesten ron Hillersohn und Stein-Bernstein. Engelmann's Arch. f. Physiol. 1896 S. 249. Dieselben fanden für normales Kälberblut die spezifische Wärme von 0,87 , für durch Zentrifugieren eingedicktes Blut 0,845 . 
von Gänse-Erythrocyten, annähernd doppelt konzentriert gegenüber Normalblut, vom spezifischen Gewicht 1,061 benutzt (N-Gehalt im Vergleich zu späteren Messungen relativ sehr hoch $=53 \mathrm{mg}$ pro Kubikzentimeter). Da die anfänglichen Messungen erheblich voneinander abwichen, wurde die Bestimmung nach zwei verschiedenen Methoden vorgenommen. Die Heizung mit dem elektrischen Strom ergab in drei Bestimmungen 0,853, 0,870 und - offenbar fehlerhaft $-0,83$ als Wärmekapazität von 1 cem Suspension. Für die spezifische Wärme der Lösung wären diese Zahlen noch durch 1,061, dem spezifischen Gewicht, zu dividieren; doch wird, da auch späterhin das Volumen, nicht das Gewicht der Lösung berücksichtigt wird, im folgenden immer mit dieser Grösse: spezifische Wärmekapazität pro $1 \mathrm{ccm}=$ spezifische Wärme $\times$ spezifisches Gewicht - gerechnet. Auf andere Weise wurde durch Einwerfen von Kupferstücken aus strömendem Wasserdampf und dem darauf erfolgenden Temperaturanstieg der Blutsuspension die spezifische Wärme derselben nach der Mischungsformel berechnet, nachdem zuvor durch Einwerfen in Wasser die spezifische Wärme der benutzten Kupferstücke bestimmt war. Für das Kupfer wurde gefunden als spezifische Wärme 0,0947, $0,0952,0,0948,0,0971$, im Mittel 0,095. Unter Benutzung von $14,33 \mathrm{~g}$ desselben ergab sich für obige Suspension als Wärmekapazität pro Kubikzentimeter in drei Messungen $0,859,0,856$ und 0,878 . Das Kupfer wurde in Spuren angegriffen, doch wird ein daraus entspringender Fehler unerheblich sein. Als Mittel aller Bestimmungen ergibt sich als Wärmekapazität pro Kubikzentimeter 0,858; im folgenden ist sie mit 0,86 gerechnet.

Über die Wärmetönung der Hämoglobingasbindungen liegen mehrere Experimentaluntersuchungen vor, die aber ausserordentlich abweichende Werte ergaben. Nach Berthelot ${ }^{1}$ ) treten für die Bindung von $1 \mathrm{mg} \mathrm{O}_{2}$ an Hämoglobin $0,48 \mathrm{~g}$ cal. auf, nach $\mathrm{Ca} \mathrm{m}$ i s $^{2}$ ) nur $0,323 \mathrm{~g}$ cal., nach Torup ${ }^{3}$ ) offenbar noch weniger. Dagegen fanden neuerdings $\mathrm{Barcroft}$ und $\mathrm{Hill}^{4}$ ) 1,75-1,98 cal. pro $1 \mathrm{~g}$ Hämoglobin, woraus sich für $1 \mathrm{mg} \mathrm{O}_{2} 0,91-1,04$, also etwa $1,0 \mathrm{cal}$.

1) Compt. rend. de l'acad. d. Scienc. t. 109 p. 778; vgl. Jules Lefèvre, Chaleur animale p. 651. 1911.

2) Biochem. Zentralbl. Bd. 7 S. 550.1908.

3) Biochem. Zentralbl. Bd. 5 S. 667. 1906.

4) Journ. of Physiol, vol. 39 p. 411. 1909/1910. 
berechnet. Noch widersprechender sind die Angaben über die Wärmetönung der $\mathrm{CO}_{2}$-Hämoglobinbindung. Während $\mathrm{Cam}$ is eine positive Wärmetönung von 0,12 cal. pro $1 \mathrm{mg} \mathrm{CO}_{2}$ findet, stellte Torup eine negative Wärmetönung von 0,5 cal. pro $1 \mathrm{mg} \mathrm{CO}$ fest.

Die einfachste Methode, diese Unsicherheit fortzuschaffen, bestand in der Durchleitung eines konstanten Sauerstoffstroms durch das Versuchsgefäss, da so die Reduktion des Oxyhämoglobins verhindert und hinsichtlich der $\mathrm{CO}_{2}$-Bindung ein stationärer Zustand geschaffen werden musste, indem die neugebildete Kohlensäure zum überwiegenden Teile entfernt wurde. Diese Messungen ergaben das unerwartete Resultat, dass die Grösse der Wärmeerzeugung dadurch nicht geändert wurde, $d$. $b$. dass sich die negative Wärmetönung der Hämoglobinreduktion und die positive der $\mathrm{CO}_{2}$-Bindung annähernd aufheben mussten. - Dass dies allerdings nicht ganz streng der Fall sein kann, zeigt schon die theoretische Überlegung, dass die Kurve der Oxybämoglobindissoziation gerade umgekehrt verläuft wie die der $\mathrm{CO}_{2}$-Bindung, indem anfänglich der Sauerstoff grösstenteils aus der umgebenden Lösung, dann zu immer grösserem Bruchteil aus dem Hämoglobin entnommen wird, während die anfänglich gebildete Kohlensäure annähernd ganz ans Hämoglobin und die Karbonate des Zellinneru gebunden wird, allmählich aber immer steigende Überschüsse in die umgebende Lösung treten.

Ausserdem wurde eine Versuchsreihe zur Bestimmung der Wärmetönung bei der Bindung bestimmter Kohlensäuremengen an Erythrocyten durchgeführt. Wenn diese auch für die Gewinnung absoluter Werte zu ungenau ist, dürfte sie doch der Grössenordnung nach zutreffend sein und sei deshalb hier angeführt, obgleich auf das Resultat kein grosses Gewicht gelegt werden soll. Die Methode war folgende: Eine NaCl-Lösung von bekanntem Gehalt an Kohlensäure wurde in ein unten mit eingeschliffenem Glasstopfen verschlossenes weites Rohr gefüllt, welches oben mit doppelt durchbohrtem Korkstopfen versehen war. In dem Korkstopfen steckte fest ein Beckmann Thermometer und lose ein schraubenförmiger Glasrührer. Diese ganze Anordnung wurde in ein weithalsiges kugliges Dewargefäss gehängt, in dem sich die Erythrocytensuspension befand, und war durch einen oben über das Thermometer gezogenen Korkstopfen in dessen Hals befestigt. Die meisten Messungen fanden nicht im Thermostaten, sondern in einem Wattekasten statt, obwohl, wie spätere Versuche zeigten, letzteres genauer gewesen wäre. Nachdem die Temperatur im Innern sich ausgeglichen hatte, wurde von aussen durch Druck auf den Glasrührer der eingeschliffene Glasstopfen aus dem Rohr herausgestossen. Infolgedessen vermischte sich die kohlensäurehaltige Innenlösung mit der Blutkörperchensuspension, und ohne irgendwelche Wärmeverluste wurde die auftretende Wärmetönung am Temperaturanstieg in den folgenden Minuten gemessen. Gelegentlich wurde auch die Blutkörperchensuspension in das 
Rohr, die Flüssigkeit aussen eingefüllt. Als Erythrocyten wurden Rinderblutkörperchen benutzt, da Gänseerythrocyten durch Atmung Störungen ergeben hätten. Die Kohlensäurelösung wurde so hergestellt, dass in geschlossenem Messzylinder eine bestimmte Menge $\mathrm{n} \mathrm{HCl}$ vorsichtig unter eine Lösung von Natriumbicarbonat geschichtet wurde, wodurch eine äquimolekulare Menge $\mathrm{CO}_{2}$ freigemacht wurde. Nach kräftigem Vermischen unter luftdichtem Verschluss des Zylinders wurde die Lösung vorsichtig in das Versuchsgefäss übergehebert. Doch fand jetzt während der Wartezeit im Dewargefäss eine dauernde geringe Entgasung statt, so dass von dem berechneten $\mathrm{CO}_{2}$-Gehalt schätzungsweise $5-10 \%$ in Abzug zu bringen sind. Eine weitere Ungenauigkeit bestand darin, dass im allgemeinen nur gegen 1 Stunde für den Temperaturausgleich gewartet wurde, was etwas knapp ist. Der Hämoglobingehalt wurde nach $\mathrm{Sahli}$ bestimmt, das Sahli'sche Hämoglobinometer durch eine maximale Sauerstoffzehrung einer abgemessenen Menge Gänseerythrocyten und nachherige Sättigung im Barcroft'schen Manometer geeicht. Sahli 100 entsprach $0,246 \mathrm{ccm} \mathrm{O}_{2}$ im Kubikzentimeter oder (da nach $\mathrm{H} \ddot{\text { üner }}$ $1,34 \mathrm{ccm} \mathrm{O}_{2}=1 \mathrm{~g}$ Hämoglobin sind) $0,184 \mathrm{~g}$ Hämoglobin. In der folgenden Tabelle sind aus den benutzten Mengen HCl-Lösung $(-10 \%)$ und der eingefüllten Flüssigkeit die Anzahl Kubikzentimeter $\mathrm{CO}_{2}$ ausgerechnet, die sich beim Vermischen auf die Blutkörperchensuspension in $240 \mathrm{ccm}$ verteilen. Für die Berechnung der Wärmetönung pro Bindung von $1 \mathrm{ccm} \mathrm{CO}_{2}$ ist in Betracht zu ziehen, dass die Kurve der $\mathrm{CO}_{2}$-Hämoglobinbindung sich auf die Tension der Kohlensäure bezieht ${ }^{1}$ ), also zum Vergleich für die Atmungsversuche, bei denen die Kohlensäure in $180 \mathrm{ccm}$ Flüssigkeit entsteht, eine um 25\% geringere Menge Koblensäure in Anschlag zu bringen ist und bei ähnlicher Hämoglobinmenge ungefähr dieselbe Wärmetönung ergeben würde. Die gebundene Menge Kohlensäure und damit die Grösse der Wärmetönung hängt von den zwei Variabeln der anwesenden Menge Hämoglobin und der Kohlensäuretension ab, bei grösseren Kohlensäuremengen wesentlich von der ersteren, weil dann der grösste Teil der Kohlensäure in der umgebenden Lösung bleibt. So ersieht man aus der folgenden Tabelle, dass die

Tabelle I.

\begin{tabular}{l|c|c|c|c|c}
\hline Nr. & $\begin{array}{c}\text { Hämoglobin } \\
\text { nach Sahli }\end{array}$ & $\begin{array}{c}\text { Berechnet } \\
\text { Hämoglobin } \\
\mathrm{g}\end{array}$ & $\begin{array}{c}\text { Berechnet } \\
\mathrm{CO}_{2} \\
\mathrm{ccm}\end{array}$ & $\begin{array}{c}\text { Temperatur- } \\
\text { anstieg } \\
0 \mathrm{C}\end{array}$ & $\begin{array}{c}\text { Berechnet } \\
\mathrm{g} / \mathrm{cal}\end{array}$ \\
\hline & 32 & 14 & 50 & 0,085 & 8,5 \\
1. & 58 & 25,5 & 20 & 0,05 & 12,0 \\
2. & 58 & 25,5 & 20 & 0,045 & 11,0 \\
3. & 46 & 20 & 16 & 0,03 & 7,0 \\
4. & 46 & 20 & 16 & 0,035 & 8,5 \\
5. & 19 & 9 & 70 & 0,026 & 6,9 \\
$\left.6 .{ }^{2}\right)$ & & & & & \\
\end{tabular}

1) Siehe Bohr, Nagel's Handbuch Bd. 1 S. 71 und 103 ff. Skand. Arch. Bd. 3 S. 66.

2) Diese Messung fand im Thermostaten von $29^{\circ}$ statt und ist, abgesehen von dem Entgasungsfehler, die genaueste. Es befanden sich $38 \mathrm{ccm}$ Blutkörperchen im Glasrohr und $220 \mathrm{ccm}$ Kohlensäurelösung aussen. 
Wärmetönung im allgemeinen von der Menge des rorhandenen Hämoglobins, bei geringen Kohlensäuremengen aber auch von der Grösse dieser abhängig ist. Übrigens kommt die auch bei grossen Kohlensäuremengen stets auftretende Lösungswärme in der hier angegebenen Versuchsanordnung nicht zur Geltung, so dass diese mit dem Fall, wo die Kohlensäure durch Atmung entsteht, nicht streng vergleichbar ist.

Bei den kleinsten Mengen Kohlensäure, die, bezogen auf eine Flüssigkeitsmenge von $180 \mathrm{~cm}$, einer Tension von $12-14 \mathrm{ccm} \mathrm{CO}$ entsprechen würden, treten also etwa $8 \mathrm{cal}$. auf. Wenn bei der Atmung annähernd $1 \mathrm{ccm} \mathrm{CO}_{2}$ für $1 \mathrm{ccm} \mathrm{O}_{2}$ auftritt, so würde also pro $1 \mathrm{mg} \mathrm{O}_{2} 0,4$ cal. für die Bindung der gleichzeitig gebildeten Menge Koblensäure entstehen müssen. Aus der obigen Erwägung sieht man aber, dass die Wärmetönung mehr als doppelt so gross sein müsste, wenn man die Barcroft'sche Zahl für die Wärmetönung der Sauerstoffhämoglobinbindung zugrunde legt und sich die positive Wärmetönung der Kohlensäurebindung gegen die negative der Hämoglobinreduktion aufhebt.

Die Kenntnis des Temperaturkoeffizienten der Atmung wurde aus methodischen Gründen gefordert, weil zwischen dem Wärmeversuch und dem Sauerstoffversuch Unterschiede von einigen Zehntelgraden schon wegen des im Dewargefässe statthabenden Temperaturanstiegs unausbleiblich waren. Ausserdem hatte diese Grösse auch ein theoretisches Interesse. Sie ergab sich für $10^{\circ} \mathrm{zu} 2,0-2,1$ zwischen $18,6^{\circ}$ und $38,1^{\circ}$.

\section{Tabelle II.}

$v$ Volumen des Absorptionsgefässes. $p$ Druckverminderung in Millimeter Manometerlösung. $t$ Temperatur des Absorptiongefässes. $m$ benutzte Menge Blutkörperchen in Kubikzentimetern. $A$ Milligramm $O_{2}$, die $10 \mathrm{ccm}$ Blutkörperchensuspension in 2 Stunden verbrauchen. $B$ Temperaturkoeffizient, umgerechnet auf $10^{\circ}$ Temperaturdifferen $\mathrm{z}^{1}$ ).

\begin{tabular}{|c|c|c|c|c|c|c|c|}
\hline $\begin{array}{c}\text { Thermo- } \\
\text { statentemp. }\end{array}$ & Zeit & $v$ & $p$ & $t$ & $m$ & $A$ & $B$ \\
\hline $\begin{array}{l}38,1^{0} \\
29,0^{\circ} \\
18,6^{\circ}\end{array}$ & $\begin{array}{l}2^{\mathrm{h}}-3^{\prime} \\
2^{\mathrm{h}} \\
2^{\mathrm{h}}+3^{\prime}\end{array}$ & $\begin{array}{l}30,7 \\
30,9 \\
29,0\end{array}$ & $\begin{array}{r}184 \\
100 \\
51\end{array}$ & $\begin{array}{l}16^{0} \\
16^{\circ} \\
16^{0}\end{array}$ & $\begin{array}{l}6,1 \\
6,3 \\
6,2\end{array}$ & $\begin{array}{l}1,29 \\
0,688 \\
0,319\end{array}$ & $\left\{\begin{array}{l}2,0 \\
2,1\end{array}\right.$ \\
\hline
\end{tabular}

1) Wenn die Atmungsgrösse bei der Temperatur $t=a$ und bei der Temperatur $t+i=a_{1}$ ist, so ist der Temperaturkoeffizient für $10^{\circ}$ nicht $=\frac{10}{i} \times \frac{a_{1}}{a}$, sondern $=\left(\frac{a_{1}}{a}\right)^{\frac{10}{i}}$. Umgekehrt ist bei einem Temperaturkoeffizienten von 2 auf $10^{\circ}$ der Zuwachs pro $0,1^{\circ}$ nicht $1 \%$, sondern $0,7 \%$. Siehe auch A. V. Hill, Journ. of Physiol. vol. 63 p. 383.1911. 
Bei den folgenden Experimenten und Berechnungen wurde so verfahren: Eine grössere Menge frisch entnommenen Gänsebluts (etwa $200 \mathrm{ccm}$ ) wurde mehrmals in $0,9 \%$ iger $\mathrm{NaCl}-\mathrm{Lösung}$ gewaschen, die Suspension in den Thermostaten von $29^{\circ}$ gehängt und bei dieser Temperatur durch Schütteln mit Sauerstoff gesättigt. Eine gemessene Menge wurde nach geeignetem Vorwärmen in das Dewargefäss gefüllt, so dass möglichst die Anfangstemperatur etwas unter der Thermostatentemperatur lag und diese überschneiden musste. Eine so genaue Regulierung gelang allerdings nicht jedesmal. Nachdem das Gefäss etwa 20 Minuten im Thermostaten gehangen batte und mehrfach umgeschwenkt war, wurde angefangen, den Temperaturanstieg zu notieren, der meist für jede Stunde oder kleinere Zeitabschnitte genau bestimmt wurde. Zur selben Zeit wurden Proben der gleichen Blutkörperchensuspension in ca. $6 \mathrm{ccm}$ fassende Atmungsgläser gefüllt, ein e Probe wurde vergiftet, eine oder mehrere andere in den Thermostaten gelegt und nach bestimmten Zeiten vergiftet. Das spezifische Gewicht wurde mit einem Aräometer bei $19^{\circ}$ oder einer nahegelegenen Temperatur bestimmt, gelegentlich auch der Stickstoff nach K Jeld ahl und der Hämoglobingehalt nach Sahli. Da die spezifische Wärmekapazität von $100 \mathrm{ccm}$ Suspension vom spezifischen Gewicht 1,061 zu 86 gefunden wurde, $0,9 \%$ ige NaCl-Lösung vom spezifischen Gewicht 1,006 aber die Wärmekapazität 100 hat, so ist z. B. die Wärmekapazität von $100 \mathrm{ccm}$ Erythrocytensuspension vom spezifischen Gewicht 1,050= $100-\frac{1050-1006}{1061-1006} \times 14=89$. Auf diese Weise wurde die Wärmekapazität der benutzten Flüssigkeitsmenge berechnet und dazu 19,5 für den Wasserwert des Kalorimeters addiert. An den abgelesenen Temperaturen wurden als Korrekturen angebracht: 1. die Gradwertkorrektur des Thermometers, die bei $29^{\circ} 1,011$ für abgelesen 1,000 betrug, 2. die Korrektur für die Differenz der Innentemperatur gegen den Thermostaten. Diese betrug für das Gefäss D IV bei Füllung mit $180 \mathrm{ccm}$ Wasser im allgemeinen 5,0, für die ersten beiden Stunden etwa 4,7, falls die Temperatur unter, 5,3\%, falls sie über dem Thermostaten lag. Bei Füllung mit Blutkörperchensuspension mussten diese Werte noch durch die Wärmekapazität der Suspension dividiert werden, weil bekanntlich die Auskühlungsgeschwindigkeit ceteris paribus der Wärmekapazität proportional ist. Für eine spezifische Wärmekapazität von 0,9 pro Kubikzentimeter 
betrug also die Auskühlung $5,5 \%$, was sich auch ziemlich gut durch Kontrollen mit Ochsenblutkörperchen bestätigen liess. (Theoretisch sind auch noch die Abweichungen der Flüssigkeitsmengen von $180 \mathrm{~cm}$ für die Auskühlung von Wichtigkeit ${ }^{1}$ ), doch waren dieselben gering und wurden vernachlässigt.) 3 . Endlich musste auf Grund des ermittelten Temperaturkoeffizienten eine Korrektur der Atmungsgrösse vorgenommen werden, indem für je $0,1^{\circ} \mathrm{C}$. Abweichung vom Thermostaten $0,7 \%$ des Gesamtausschlages addiert oder subtrahiert wurde. Über die Sanerstoffmethode ist das Nötige schon oben gesagt.

Der Temperaturanstieg erfolgt unter Berücksichtigung aller Korrekturen für die erste Zeit annähernd gleichmässig; doch zeigt sich in Übereinstimmung mit genauen Sauerstoffbestimmungen nach 2-3 Stunden ein allmähliches geringes Absinken, das auf eine Schädigung durch die NaCl-Lösung oder durch Anhäufung von $\mathrm{CO}_{2}$ bezogen werden kann. Kurve I auf Tafel II zeigt die Wärmeproduktion für 8 Stunden. Sie entstammt dem Versuch 7. Ähnliche Kurven ergaben sich bei den übrigen Versuchen.

Der korrigierte Temperaturanstieg beträgt in diesem Fall für die 1 . Stunde $0,099^{\circ}$ )

\begin{tabular}{|c|c|c|c|}
\hline & 2. & & $0,096^{\circ}$ \\
\hline & & $n$ & $0,096^{\circ}$ \\
\hline & 4. & & $0,085^{0}$ \\
\hline & $" 5$. & " & $0,077^{\circ}$ \\
\hline & 6. & $n$ & $0,070^{\circ}$ \\
\hline & 7 & $"$ & $0,0655^{\circ}$ \\
\hline & $\pi 8$. & , & $0,066^{\circ}$ \\
\hline
\end{tabular}

Bei anämischem Blut ist eine so lange Fortsetzung des Experiments nicht möglich, weil es auf den gleichen Sauerstoffvorrat im Hämoglobin eine mehr als doppelt so grosse Atmung zeigt. Bei nicht anämischem Blut betrug der Temperaturanstieg bei ungefährer Konzentration von Normalblut $0,060-0,065^{\circ}$ pro Sturide oder 11-12 cal. auf $185 \mathrm{ccm}$. Pro Kubikzentimeter werden also etwa 0,06 cal. gebildet. Anderseits enthält $1 \mathrm{ccm}$ Erythrocytensuspension von Normalblutkonzentration $20-25 \mathrm{mg} \mathrm{N}$. Auf $140 \mathrm{mg} \mathrm{N}$ entstehen also ungefähr 0,4 cal. pro 1 Stunde, halb so viel wie bei unbefruchteten Seeigeleiern.

1) Vgl. Hill, Journ. of Physiol. vol. 53 p. 265.1911.

2) Berechnet aus 50 Minuten. 
Die auf S. 171 befindliche Tabelle III enthält, abzüglich einiger ungenauer Vorversuche, alle zur Bestimmung des Quotienten gemachten Experimente: zwölf Bestimmungen für unbeeinflusste Atmung, fünf für Atmung unter Durchleitung eines Sauerstoffstroms und zwei für Atmung in $3 \%$ igem Äthylurethan.

Ausser den angeführten Versuchen fand noch ein Vergleich der Wärmebildung in der gleichen Blutkörperchensuspension mit und ohne Durchleitung von Sauerstoff statt. Zuerst wurde der Temperaturanstieg 2 Stunden $30 \mathrm{Min}$. im geschlossenen Gefäss verfolgt und betrug für diese Zeit $0,091^{\circ}=0,0365$ in 1 Stunde. Dann wurde der Sauerstoff plötzlich zugelassen und strömte langsam für die nächsten 2 Stunden 20 Min. durch das Versuchsgefäss hindurch. In dieser Zeit betrug der Anstieg $0,086^{\circ}$, oder $0,037^{\circ}$ in 1 Stunde, also ebensoviel. Ausserdem müsste sich ein Überschuss positiver Wärmetönung der Oxyhämoglobinbildung beim plötzlichen Einleiten von Sauerstoff in Blut, das längere Zeit geatmet hat, bemerkbar machen. In Wirklichkeit treten hierbei stets umgekehrt geringe Temperatursenkungen ein. Doch ist ein Schluss hieraus nicht zu ziehen, weil durch das plötzliche Aufschäumen der Flüssigkeit Komplikationen entstehen.

Dass bei der Atmungshemmung durch Narkotika der gleiche kalorische Quotient vorliegt wie bei gewöhnlicher Atmung, musste nach ähnlichen Versuchen am Seeigelei erwartet werden, dürfte aber doch Interesse erwecken. Diese Tatsache zeigt, dass an Stelle der gehemmten Oxydation nicht etwa andere energetische Prozesse einsetzen, dass also die Hemmung sich nicht auf den Sauerstoffkonsum, sondern auf den Energieumsatz der Zelle bezieht.

\section{Wärmetönungen im Säugetierblut.}

Es wurden zahlreiche Messungen mit Blut und Blutbestandteilen von verschiedenen Säugetieren angestellt, hauptsächlich, um zu entscheiden, ob hier Wärmebildung und Sauerstoffverbrauch ebenfalls parallel gehen, also ein bestimmter kalorischer Quotient nachweisbar ist oder nicht. Es zeigte sich dabei, dass gelegentlich Wärmetönungen auftreten, für die ein erheblich zu kleiner Sauerstoffverbrauch stattfindet. Das Ungekehrte, Sauerstoffverbrauch mit zu geringer Wärmetönung, wurde nicht gefunden bzw. liegen einzelne solche Feststellungen an Ungenauigkeiten im Beginn der Wärme- 
Über Wärmetönungen chemischer Prozesse in lebenden Zellen.

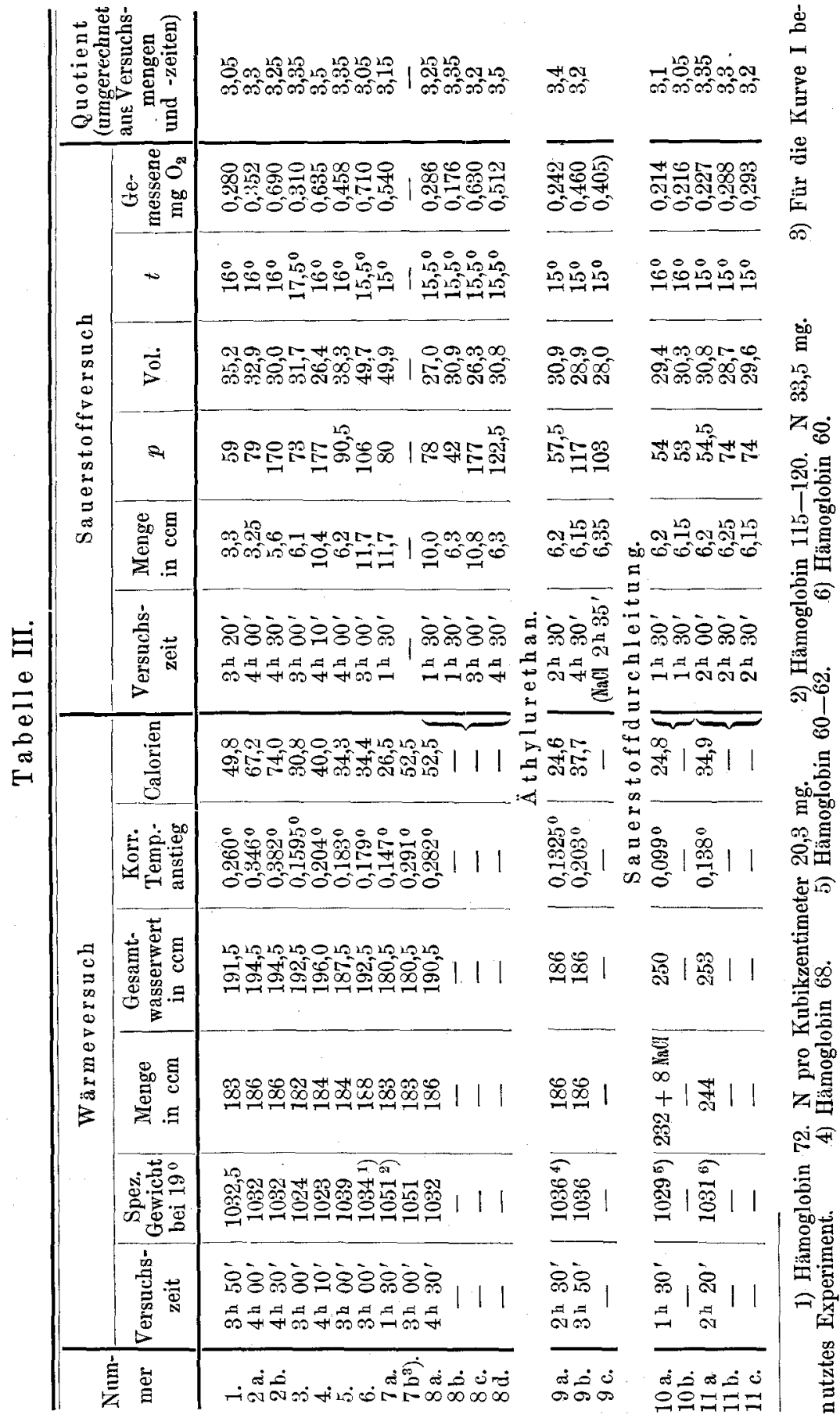


versuche. Anderseits fand sich wiederholt auch ein gutes Zusammentreffen beider Werte, und des öfteren sind Abweichungen schwer von Versuchsfehlern zu unterscheiden, weil, wie im hiesigen Institut gezeigt wurde, einzelne atmende Elemente durch die Art der Behandlung, z. B. Schütteln, geschädigt werden und in der Zeit stark veränderlich sind ${ }^{1}$ ), aber beide Parallelversuche hierin nie genau übereinstimmen. Wenn man noch in Betracht zieht, dass die atmenden Bestandteile bei verschiedenen Säugetieren eine verschiedene Rolle spielen und enorme Blutmengen zur Anstellung der Versuche erforderlich sind (für e in en Versuch musste ein grosses oder zwei mittelgrosse Kaninchen, oder ein mittelgrosser Hund ganz ausgeblutet werden), so mag es verständlich sein, dass die hier angestellten Versuchsreihen abgebrochen wurden, ehe über alle Punkte Klarheit erzielt worden ist. Wärmetönungen, für die keine entsprechende Sauerstoffzehrung gefunden wurde, wurden gelegentlich im Blute aller untersuchten Tiere gefunden; doch fand sich beim Kaninchen zumeist annähernd Übereinstimmung beider Werte, so dass gelegentliche Abweichungen auf den angeführten Versuchsungenauigkeiten beruhen können. Diese Wärmetönungen ohne Sauerstoffzehrung scheinen an den Erythrocyten zu hängen, denn sie fanden sich auch in gewaschenen Blutkörperchen, das Plasma allein dagegen gab sie nicht. Die Atmung der Erythrocyten ist beim Menschen und Kaninchen bestimmt von O. Warburg ${ }^{2}$, die der Blutplättchen beim Hund und Kaninchen von M. Onaka ${ }^{3}$.

Im folgenden ist eine Übersicht über die Versuche bei den einzelnen Tierarten gegeben. Bei der Berechnung der gebildeten Kalorien ist als spezifische Wärme von Normalsäugetierblut der Wert von Hillersohn und Stein-Bernstein $=0,87$ zugrunde gelegt, was einer Wärmekapazität des Kubikzentimeters Blut von 0,91 entspricht, für zusammenzentrifugierte Blutkörperchen die spezifische Wärme $0,84=0,89$ Wärmekapazität pro Kubikzentimeter. Als Gefässe dienten neben den bisherigen noch ein dem Gefäss D II genau entsprechendes Gefäss DI von gleichem Rauminhalt und gleichem Abkühlungskoeffizienten. Wo das in $0,002^{\circ}$ geteilte Thermometer benutzt wurde, wurde der Wasserwert um 2,0 wegen des grossen Quecksilbergefässes des Thermometers erhöht.

1) Siehe Onaka, Zeitschr. f. physiol. Chemie Bd. 71 S. 193.

2) Zeitschr. f. physiol. Chemie Bd. 59 S. 112.

3) Zeitschr. f. physiol. Chemie Bd. 71 S. 193. 


\section{Kaninchen.}

1. $165 \mathrm{ccm}$ Blut eines sehr grossen Kaninchens in $10 \mathrm{ccm}$ Hirudinaufschwemmung aufgefangen, 10 Minuten nach Ausblutung des Kaninchens in D IV eingefüllt. Beginn der Messung 5 Minuten später (unter diesen Umständen ist die erste Stunde um mehrere Tausendstelgrad ungenau).

Temperaturanstieg: $\quad$ erste Stunde $0,045^{\circ}$, zweite $\Rightarrow 0,051^{\circ}$, dritte $" 0,049^{\circ}$.

Wasserwert 180. Im ganzen sind in 3 Stunden 26 cal., pro Kubikzentimeter unverdünnten Blutes in 3 Stunden 0,163 cal. erzeugt, nicht viel weniger wie im Gänseblut.

2. $210 \mathrm{ccm}$ Blut von zwei mittelgrossen Kaninchen in $25 \mathrm{ccm}$ Hirudinaufschwemmung aufgefangen, $189 \mathrm{ccm}$ eingefüllt in DIV. Versuch begonnen eine halbe Stunde nach der Entblutung.

Temperaturanstieg:

erste Stunde . . . . $0,029^{\circ}$,
zweite $\Rightarrow . \cdot . \cdot . \cdot 0,031^{\circ}$,
dritte und vierte Stunde je $0,0345^{\circ}$,
fünfte Stunde zur Hälfte . $0,017^{\circ}$.

Wasserwert 198. In 41/2 Stunden 28,2 cal. erzeugt. Pro $1 \mathrm{ccm}$ unverdünnten Blutes etwa 0,135 cal. Der Sauerstoffversuch ist wegen zu grosser Kohlensäuremenge ungenau, ergibt aber der Grössenordnung nach den normalen Quotienten.

3. Blut eines mittelgrossen Kaninchens unter Nachspülen von NaCl-Lösung in Hirudin aufgefangen. Verdünnt ungefähr um die Hälfte. Das Blut gerinnt während des Versuchs.

Temperatur: erste Stunde . . . . 0,014 ${ }^{\circ} \mathrm{C}$,

zweite Stunde. . . . . $0,012^{\circ} \mathrm{C}$.,

vierte und fünfte Stunde je $0,020^{\circ} \mathrm{C}$.,

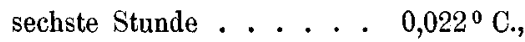

D IV $164 \mathrm{ccm}$.

siebente Stunde.... $0,019^{\circ} \mathrm{C}$.

4. $95 \mathrm{ccm}$ eines mittelgrossen Kaninchens in Na-Citrat aufgefangen und mit $105 \mathrm{ccm} \mathrm{NaCl-Lösung} \mathrm{versetzt.} 186 \mathrm{ccm}$ in D IV gefüllt.

Erste Stunde . . . 0,011 ${ }^{\circ}$,

zweite Stunde . . . 0,008,

dritte und vierte Stunde je $0,017^{\circ}$.

Im ganzen sind in 4 Stunden 10,3 cal. gebildet; Sauerstoffverbrauch $\left(p=16 \mathrm{~mm}, v=31 \mathrm{ccm}, t=16^{\circ}\right.$, Menge $=16,2 \mathrm{ccm}$, Zeit 4 Stunden) um ein Drittel zu gering, so dass $Q=5$ entstünde. Wärmeerzeugung in 4 Stunden pro Kubikzentimeter unverdünnten Blutes 0,12 cal.

5. $152 \mathrm{ccm}$ Blut in $20 \mathrm{ccm}$ Natriumcitrat aufgefangen. Blutkörperchen in $\mathrm{NaCl} \cdot$ Lösung gewaschen, auf $225 \mathrm{ccm}$ aufgefüllt. $180 \mathrm{ccm}$ davon (D IV) ergeben einen Temperaturanstieg von $0,042^{\circ}$ in 3 Stunden $=0,014^{\circ}$ pro 1 Stunde, vierte Stunde $0,021^{\circ}$, zusammen in 4 Stunden $0,063^{\circ}$. Wasserwert $193.12,15$ cal. (pro $1 \mathrm{ccm}$ unverdünnten Blutes $0,10 \mathrm{cal}$. in 4 Stunden). Sauerstoffverbrauch ( $p=50,5$, $v=48,8, t=15^{\circ}, m=11,7$, Zeit 4 Stunden) gibt den Quotient 2,5, annähernd 
„normal“, zumal bei sehr kleinen Temperaturanstiegen die erste Stunde stets einen zu kleinen Wert gibt.

6. Geschlagenes Blut. $130 \mathrm{~cm}$ Blut $+170 \mathrm{ccm} \mathrm{NaCl-Lösung.} \mathrm{Erste} \mathrm{Stunde}$ $0,008^{\circ}$, zweite Stunde $0,007^{\circ}$ dritte und vierte Stunde je $0,0155^{\circ} .185 \mathrm{ccm}$ Blut. Wasserwert 193. $1 \mathrm{ccm}$ unverdünnten Blutes in 4 Stunden 0,09 cal.

Bezüglich der absoluten Grösse der Ausschläge ist zu sagen, dass die erheblich höhere Wärmetönung bei in Hirudin und Natriumcitrat aufgefangenem gegenüber gewaschenem und geschlagenem Blut jedenfalls durch die Atmung der Blutplättchen bedingt ist. Die Abweichung des kalorischen Quotienten vom normalen Wert könnte angesichts der Kleinheit der Ausschläge auf ein verschiedenes Verhalten der Blutplättchen in den Parallelversuchen bezogen werden.

\section{Mensch.}

7. Blut eines Neplritikers, $1 / 2$ Stunde nach der Venesektion eingefüllt in D IV. (Anämisches Blut, Sahli 45), Na.Citrat.

erste Stunde. . . . . . . . . . . 0,09\%, zweite, dritte und vierte Stunde zusammen $0,037^{\circ}$ je $0,012^{\circ}$, fünfte und sechste Stunde zusammen $0,021^{\circ}$. . je $0,015^{\circ}$.

Die ersten 4 Stunden 30 Minuten (1. $-4^{1 / 2}$. Stunde) $0,050^{\circ}$, die letzten 4 Stunden 30 Minuten (2/2.-6. Stunde) $0,056^{\circ}$ entsprechend 10,0 und 11,1 cal. $190 \mathrm{ccm}$, Wasserwert 199. In den letzten 4 Stunden 30 Min. $p=27, v=30$. $t=16, m=6,1 ; 0,11 \mathrm{mg} O_{2}$, oder $3,6 \mathrm{mg} \mathrm{O}_{2}$ in der Menge des Wärmeversuchs, $Q=3,1$.

8. Arteriosklerose. Blutkörperchen in Natriumcitrat aufgefangen, gewaschen, aufgefüllt aufs ursprüngliche Volumen, $1^{1 / 2}$ Stunden nach Venesection eingefüllt. In 3 Stunden $30 \mathrm{Min}$. Temperaturveränderung $+0,000^{\circ}$.

9. Nephritis. Blut $3 / 4$ Stunde nach Venesektion eingefüllt. Na-Citrat.

Temperatur-Anstieg $1 / 2$ Stunde . . . 0,034 ${ }^{\circ}$,

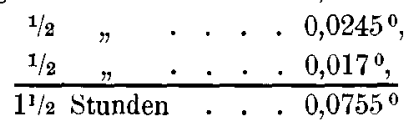

Hämoglobin S a hli $73,190 \mathrm{ccm}$. Wasserwert 192 . In 11/2 Stunden 14,5 cal. erzeugt; in unverdünntem Blut per cem 0,055 cal. in 1 Stunde, d. h. ebensoviel wie im Gänseblut. Sauerstoffversuch $\left(p=13, v=30, t=15^{0}, m=6,2\right.$, Zeit $1 \frac{1 / 2}{2}$ Stunden) gibt noch nicht ein Drittel des erforderlichen Wertes.

10. Pneumonie. Blut in Natriumcitrat aufgefangen, $255 \mathrm{ccm}$. Zweimal in NaCl-Lösung gewaschen, auf $230 \mathrm{ccm}$ gebracht, eingefüllt $1 \frac{1 / 2}{2}$ Stunden $p . v$.

1 Stunde 10 Minuten $0,035^{\circ}$, pro 1 Stunde $0,030^{\circ}$;

dann 1 Stunde 20 Minuten $0,032^{\circ}$, pro 1 Stunde $0,024^{\circ}$.

\section{Hund.}

11. $600 \mathrm{ccm}$ Blut in Na-Citrat aufgefangen; davon $190 \mathrm{~d} / 4$ Stunde nach der Entblutung eingefüllt in D IV.

In 2 Stunden 40 Minuten $0,044^{\circ}=0,018^{\circ}$ pro 1 Stunde. 
1 Stunde 50 Minuten $0,089^{\circ}=0,0205^{\circ}$ pro 1 Stunde. $190 \mathrm{ccm}$. Hämoglobin 75. Wasserwert 194. 0,021 cal. pro $1 \mathrm{ccm}$ in 1 Stunde.

Die übrigen $410 \mathrm{ccm}$ eingedickt auf $238 \mathrm{ccm}$ Blutkörperchen geben in 2 Stunden 50 Minuten $0,107^{\circ}$ oder $0,038^{\circ} \mathrm{C}$. in 1 Stunde. (Hämoglobin 115; D II. Wasserwert 237. $0,0375 \mathrm{cal}$. pro $\mathrm{cm}$ in 1 Stunde).

Dann $3 \mathrm{mal}$ in $\mathrm{NaCl}-\mathrm{Lösung}$ gewaschen: in 1 Stunde $0,033^{\circ}$.

Umgerechnet auf die Konzentration der Blutkörperchen des Normal blutes ergibt sich für die eingedickten Blutkörperchen im Serum ebenfalls pro $1 \mathrm{ccm} 0,0215 \mathrm{cal}$. Die Wärmeproduktion findet also ausschliesslich in den Blutkörperchen statt.

12. Hundeblut in Hirudin aufgefangen $(165 \mathrm{ccm}$ Blut +15 Hirudin) gibt pro 1 Stunde $0,030^{\circ}$. Nach häufigem Zentrifugieren schliesslich nur noch $0,0215^{\circ}$ pro 1 Stunde.

Alle diese gefundenen Wärmetönungen dürften grösstenteils auf Rechnung der Atmung der relativ kernreichen Hundeerythrocyten zu setzen sein.

\section{Rind.}

Gewaschene Ochsenblutkörperchen lassen im allgemeinen nicht die geringste Wärmebildung erkennen. Dagegen zeigt frisches geschlagenes Ochsenblut eine steil abfallende Wärmebildung, die teilweise auf Blutplättchenatmung beruhen könnte.

13. Defibriniertes frisches Ochsenblut gibt (D II) erste Stunde $0,028^{\circ}$, zweite Stunde $0,014^{\circ}$, dritte und vierte Stunde je $0,008^{\circ}$. 1 Stunde.

14. D II. Temperaturanstieg in $3^{1 / 2}$ Stunden $0,0595^{\circ}$ oder $0,0165^{\circ}$ pro

15. D IV. Frisches defibriniertes Ochsenblut.

1. Stunde . . 0,030 dann $1^{1 / 2}$ Stunden $0,021^{0}$ oder $0,014^{\circ}$ pro 1 Stunde, dann 2 Stunden $0,022^{\circ}$ oder $0,011^{\circ}$ pro 1 Stunde.

\section{Die Energieverhältnisse bei Sauerstoffabschluss.}

Es wird allgemein angenommen, dass bei höherer Temperatur der Energieumsatz Bedingung des Lebens von Zellen ist. Bei Abwesenheit von Nährstoffen erschöpft die Zelle zunächst ihr eigenes Reservematerial und geht dann zugrunde. Bei Abwesenheit von Sauerstoff nimmt man auch für die aeroben Zellen an, dass dieselben, solange sie den Abschluss von Sauerstoff ertragen, ihren Energiebedarf aus Spaltungsvorgängen decken und auch ohne Sauerstoffaufnahme Kohlensäure ausscheiden. Daher wird allgemein ein Versiegen der Kohlensäureausscheidung als Zeichen des Todes angesehen. "Wird lebenstätigen Aeroben durch Wasserstoff oder Evakuation der Sauerstoff entzogen, so fahren sie zunächst fort, Kohlensäure zu produzieren, und das Erlöschen dieser Exhalation ist ein sicheres Zeichen des Todes, " sagt Pfeffer ${ }^{1}$ ).

1) Pflanzenphysiologie Bd. 1 S. 543. 1897.

Pflüger's Archiv für Physiologie. Bd. 146. 
Eigene : Versuche an zwei verschiedenen Objekten; den kernhaltigen Erythrocyten der Gans und dem obligat aeroben Vibrio Metschnikoff "werfen auf die energetische Seite des Problems ein merkwürdiges Licht. Es liessen sich zwei Tatsachen feststellen, deren Vereinigung Interesse erwecken dürfte. 1. Durch $\mathrm{mehr}-$ stündigen Abschluss von Sauerstoff bei $29^{\circ}$ wird der Vibrio Metschnikoff für die darauf folgende Zeit weder in der Grösse der Atmung noch im Wachstumsvermögen nachweisbargeschädigt, die Gänse-Erythrocyten in der Atmung nur unerheblich: 2. Während der Zeit des Sauerstoffabschlusses isteine Wärmebildung, also ein Energieumsatz, nicht nachweisbar. Die Genauigkeitsgrenze der Versuche für diese letztere Feststellung ist bei den Erythrocyten aus methodischen Gründen dergestalt, dass wenige Prozent der normalen Wärmebildung der Beobachtung hätten entgehen können; auch bezieht sich die Feststellung bei diesen nur auf die zweite Hälfte der Zeit des Sauerstoffabsehlusses. Bei den Versuchen mit Vibrio Metschnikoff war die Genauigkeit erheblich grösser, so dass hier keinesfalls $1 \%$ des gewöhnlichen Energieumsatzes erreicht wurde. 3. sei noch hervorgehoben, dass die spätere Atmung. ceteris paribus nicht grösser ist als vorher, dass also die Verbrennungen für die Zwischenzeit nicht etwa nachgeholt werden. Bei Bakterien ist die Atmung bei Beginn des Wiederzutritts von Sauerstoff ebenso gross wie vorher zum Schluss beim Absperren, steigt aber dann dem Wachstum entsprechend an. Während des Sauerstoffabschlusses hat ein Wachstum also nicht stattgefunden, dagegen setzt dies unmittelbar nach Zutritt von Sauerstoff wieder ungeschwächt ein.

Bei den Vogel-Erythrocyten wurde so verfahren, dass die Blutkörperchensuspension nach vorangegangener Atmung maximal mit der Wasserstrahlpumpe ausgepumpt und sofort Paraffin überschichtet wurde. Das Blut wurde dann in das Dewargefäss unter Paraffin übergehebert und noch einige Zeit sich selbst überlassen, um etwaige Spuren von Sauerstoff durch Atmung fortzuschaffen. Da bei der Überheberung die Temperatureinstellung nicht genau $z u$ regulieren war, fanden Abweichungen um einige Zehntelgrade vom Thermostaten statt. Hierdurch und infolge einer empfindlichen Störung des. Temperaturausgleichs durch das oben schwimmende Paraffin erklären sich die in den beiden ersten Fällen über die normale Korrektur 
noch stattgehabten Temperatursenkungen. Wie gesagt, beziehen sich die Messungen nur auf die zweite Hälfte der Zeit des Sanerstoffabschlusses, weil in der ersten die verschiedensten Manipulationen erforderlich waren und eine genaue Temperatureinstellung im Thermostaten abgewartet werden musste. Eine anfängliche Wärmebildung musste hier also der Feststellung entgehen. Alle diese Umstände lagen bei den Versuchen mit Bakterien erheblich besser.

Tabelle IV.

Gunse-Erythrocyten.

\begin{tabular}{|c|c|c|c|c|c|c|}
\hline \multirow{2}{*}{ Nr. } & \multicolumn{2}{|c|}{ Atmung vorher } & \multicolumn{2}{|c|}{ Sauerstoff abschluss } & \multicolumn{2}{|c|}{ Atmung nachher } \\
\hline & Zeit & $\begin{array}{c}\text { korr. } \\
\text { Anstieg }\end{array}$ & $\begin{array}{c}\text { Zeit des } \\
\text { Abschlusses }\end{array}$ & $\begin{array}{l}\text { korr. Tem- } \\
\text { peratur in der } \\
\text { Messungezeit }\end{array}$ & Zeit & $\begin{array}{c}\text { korr. } \\
\text { Anstieg }\end{array}$ \\
\hline 1. & - & nicht gemessen & $5 \mathrm{~h} \cdot 10^{\prime}$ & $-0,010^{\circ}$ & $1^{\text {h }} 20^{\prime}$ & $0,099^{\circ}$ \\
\hline 2. & $2^{\text {h }} 40^{\prime}$ & $0,332^{0}$ & 3 h $30^{\prime}$ & $-0,012^{\circ}$ & & $\begin{array}{r}0,154^{0} \\
\text { (Rattarienin }\end{array}$ \\
\hline 3. & ${ }_{(1 \mathrm{~h}}^{2 \mathrm{~h}} 50^{\prime}$ & $\begin{array}{l}0,189^{\circ} \\
0,067)\end{array}$ & $2^{\mathrm{h}}$ & $0,000^{\circ}$ & $\operatorname{li}_{(1 \mathrm{~h}}^{\mathrm{h}} 20^{\prime}$ & $\begin{array}{l}0,081^{\circ} \\
\left.0,061^{\circ}\right)\end{array}$ \\
\hline
\end{tabular}

Mehrere Kubikzentimeter einer dichten Bakterienemulsion, die auf Agarplatten gezüchtet und in Bouillon aufgenommen war, wurde mit in Kochsalzpeptonlösung ( $1 \%$ Pepton) gewaschenen Ochsenblutkörperchen versetzt und diese Mischung in das Dewargefäss gehängt. Wenn der gesamte Sauerstoff aus dem Hämoglobin veratmet ist, so steht den Bakterien keiner mehr zur Verfügung, zumal wenn oben eine Parafinschicht gegen die Luft abschliesst. Indes konnte, falls das Gefäss bis ganz oben gefüllt war, diese auch fortgelassen werden, weil die minimalen Sauerstoffspuren, die dann durch Diffusion in den schmalen Spalt zwischen Thermometer und Gefässhals hineingelangen können, ganz ausser Betracht kommen. Wenn dann nach mehreren Stunden das Gefäss entleert wird, die Flüssigkeit kräftig an der Luft geschüttelt und wieder zurückgefüllt wird, so ist nunmehr wieder genügend Sauerstoff für die Atmung vorhanden. Dabei zeigt sich folgendes: Im ersten Abschnitt steigt die Temperatur rapide, und zwar wie sich für jeden kleinen Zeitabschnitt (z. B. von 10 Min.) zeigen lässt, mit wachsender Geschwindigkeit an, letzteres wegen der stattfindenden Vermehrung der Bakterien. Dieser rapide Anstieg lässt innerhalb einiger Minuten schnell nach und ist nach kurzer Zeit ganz erloschen. ${ }^{\text {) }}$ )

1) Während dieser Anfangszeit könnten natürlich auch anaerobe. Umsetzungen stattfinden. Es dauert etwa 1/2 Stunde von dem Nachlassen des Temperaturanstiegs bis zur Konstanz der Temperatur. 
Von jetzt an für mehrere Stunden verändert sich die Temperatur annähernd so viel, als der berechneten Korrektur für den Thermostaten entspricht. Diese Genauigkeit ist um so grösser, je kleiner die Abweichung vom Termostaten ist, besonders wenn kein Paraffin aufgeschichtet ist. In einem Falle (auf Kurve II Taf. II abgebildet), betrug die gemesseneVeränderung in 3 Stunden $+0,013^{\circ}$, während sich als Korrektur für diese Zeit 0,010 bis $0,012^{\circ}$ berechnet, also nur $0.002^{\circ}$ Unterschied. Dabei betrug der Anstieg für die Zeit der Atmung vorher in 1 Stunde $0,392^{\circ}$. Davon in den letzten 10 Minuten $0,069^{\circ}$. Hinterher verging $\mathbf{1} / \mathbf{2}$ Stunde mit Schütteln und Neueinstellen der Temperatur, während welcher Zeit bereits ein Wachstum stattfinden konnte. Deshalb betrug der Anstieg in den ersten 10 Minuten der Messung nachher bereits korr. $0,081^{\circ}$; im ganzen in 45 Minuten $0,372^{\circ}$. Jetzt wiederholte sich dasselbe. Der Sauerstoff versagte und die Temperatur blieb konstant. Wenn in den 3 Stunden der Sauerstoffentziehung der korr. Temperaturanstieg $0,011^{\circ}$ betragen hätte, so wäre er nur $1 \%$ desjenigen bei Sauerstoffgegenwart; tatsächlich betrug er aber nur $+0,002^{\circ}$ (innerhalb der Fehlergrenze).

In einem zweiten Versuch wurde so verfahren, dass zuerst die Bakterienaufsehwemmung in Peptonkochsalz ohne Blutkörperchen unter Überschichten von Paraffin - eingefüllt wurde. Der vorhandene Sauerstoff wird hier sofort restlos aufgezehrt (bei Zusatz von Methylenblau schlägt die Lösung aus Blau in Weiss um, und nach dem Winkler-Verfahren ist keine Spur Sauerstoff mehr nachweisbar). Die Lösung wurde 2 Stunden so gehalten. Innerhalb der 1 stündigen Messungszeit fiel die Temperatur korr. um $0,007^{\circ}$. Dies liegt an der durch das Paraffin bewirkten Unregelmässigkeit. Etwa die Hälfte der benutzten Bakterienaufschwemmung, mit Blutkörperchen versetzt, ergibt in 1 Stunde nachher $0,126^{\circ}$ Temperaturanstieg. Die ganze Menge hätte also $0,25^{\circ}$ ergeben, während die Veränderung in 1 Stunde bei Sauerstoffabwesenheit $-0,007^{\circ}$ betrug. (Übrigens fanden in allen Fällen nur Blutkörperchen Verwendung, die für sich keine Wärmebildung zeigten.)

Ein dritter Versuch entsprach dem ersten. Nur war er wegen der Überschichtung von Paraffin und einem sehr starken Abweichen der Temperatur viel ungenauer. In 80 Minuten betrug der korr. Temperaturanstieg $0,517^{\circ}$. Davon in den letzten 10 Minuten $0,074^{\circ}$. Darauf fiel die Temperatur in 3 Stunden 20 Minuten um $0,028^{\circ}$, während sie entsprechend der Korrektur um etwa $0,045^{\circ}$ hätte fallen 
müssen. Nach weiteren 20 Minuten wurde die Lösung wieder an der Luft geschüttelt. Jetzt betrug der Temperaturanstieg in 40 Minuten $+0,337^{\circ}$.

Tabelle V.

Bakterien. ${ }^{1}$ )

\begin{tabular}{|c|c|c|c|c|c|c|}
\hline \multirow[b]{2}{*}{ Nr. } & \multicolumn{2}{|c|}{ Atmung vorber } & \multicolumn{2}{|c|}{ Sauerstoffabschluss } & \multicolumn{2}{|c|}{ Atmung nachher } \\
\hline & Zeit & $\begin{array}{c}\text { korr. } \\
\text { Anstieg }\end{array}$ & $\begin{array}{c}\text { Zeit des } \\
\text { Abschlusses }\end{array}$ & $\begin{array}{c}\text { korr. } \\
\text { Teraperatur } \\
\text { in dieser Zeit }\end{array}$ & Zeit & $\begin{array}{c}\text { korr. } \\
\text { Temperatur }\end{array}$ \\
\hline $\begin{array}{l}1 \\
2\end{array}$ & $1^{\text {h }} 00^{\prime}$ & $0,392^{\circ}$ & $\begin{array}{l}\begin{array}{lll}3 \text { h } & 00^{\prime} \\
2 \text { b } & 00^{\prime}\end{array}\end{array}$ & $\begin{array}{l}+0,002^{\circ} \\
-0,007^{\circ}\end{array}$ & 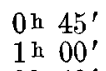 & $\begin{array}{l}0,372^{0} \\
0,25^{0}\end{array}$ \\
\hline 3 & 1 h $20^{\prime}$ & $0,517^{\circ}$ & $3 \mathrm{~h} 20^{\prime}$ & $+0,015^{\circ}$ & 0 h $40^{\prime}$ & $0,337^{\circ}$ \\
\hline
\end{tabular}

1) Die Messungen des kalorischen Quotienten der Sauerstoffatmung des Vibrio Metschnikoff sind unter dem Titel „Über den Energiewechsel von Bakterien" in den Sitzungsberichten der Heidelberger Akademie der Wissenschaften (1912) publiziert. Die wichtigsten Ergebnisse dieser Arbeit sind folgende: Bei Aufschwemmung der Vibrionen in Kochsalz $(0,5 \%$ ige)-Pepton ( $1 \%$ ige) lösung unter Zugabe gewaschener Rindererythrocyten ergab sich im Mittel von neun Wärmeund zwölf zugehörigen Sauerstoff bestimmungen der kalorische Quotient 3,95; im Mittel von fünf Wärme- und sieben zugehörigen Sauerstoffmessungen, in denen durch Zufügung von 0,5-0,7\% Isobutylurethan Atmung und Wachstum auf etwa die Hälfte herabgesetzt war, ebenfalls 3,95. Dies bestätigt die obige Feststellung, dass für die Aufhebung der Sauerstoffatmung durch Narkotika keine andern Energie liefernden Prozesse eintreten. Da die so ausgeführten Versuche eine sehr grosse Fehlerbreite zeigten, etwa $25 \%$ (also die Genauigkeit der einzelnen Versuche nur 10-15\% betrug), ausserdem das Milieu für die Vibrionen ungünstig war, wurde als Nährlösung eine Mischung von $5 \%$ Bouillion, 0,4\% Asparagin, $0,2 \%$ Kaliumbiphosphat, $0,5 \% \mathrm{NaCl}$, alkalisch gegen Phenolphthalein, in doppelt destilliertem Wasser benutzt, in der ein rapides Wachstum stattfand. Blutkörperchen wurden nicht zugegeben, sondern die Lösung maximal mit Sauerstoff gesättigt und der Sauerstoffverbrauch nach Winkler titriert. Es ergab sich in diesem veränderten Milieu in sieben Parallelversuchen ein cal. Quotient von 4,1, bei einer Fehlerbreite von weniger als $10 \%$. Endlich gelang es, unter Benutzung der gleichen Methode, den kalorischen Quotienten bei aufgeh obenem Wachstum zu bestimmen. Bei unzureichender Zufuhr von Nährstoffen, d. h. bei Benutzung von nur 0,5\% Bouillion und Weglassung von Asparagin, findet nämlich bei Fortbestand der Atmung keinerlei erkennbare Vermehrung mehr statt, sondern der Sauerstoffverbrauch in der Zeiteinheit bleibt für mehrere Stunden annähernd konstant, bzw. sinkt ganz langsam ab. In allen Fällen dieser Art fand sich ein höherer kalorischer Quotient, gegen 4,5; doch fielen einige Messungen aus unbekannten Gründen ganz heraus. Als gemeinsames Resultat ergibt sich, dass bei Bakterien jedenfalls zu den Oxydationsvorgängen noch andere wärmebildende Reaktionen hinzukommen, die zwar durch den Oxydationsprozess angeregt werden, aber nicht selbst chemisch ihm angehören, etwa Neutrali- 


\section{IV. Über Wärmetönungen beim Eindringen von atmungs- hemmenden Substanzen in lebende Zellen.}

0. Warburg hat gefunden, dass die oxydationshemmenden Substanzen sich hinsichtlich ihrer Wirkungsweise in mehrere Gruppen teilen lassen. Die indifferenten Narkotika wirken nicht unmittelbar nach ihrer chemischen Konstitution, sondern nach dem sogenannten Gesetz der "homologen Reihen". Demgegenüber fand Warburg zwei andere davon deutlich unterschiedene Gruppen, deren Wirkungsstärke direkt mit ibrer chemischen Konstitution in Verbindung steht, erstens die Aldehyde und zweitens die substituierten Ammoniake. Die Aldehyde wirken ausserordentlich viel stärker, als es ihrem Teilungsverhältnis in den Lipoiden entspricht und ausserdem durchaus nicht stärker beim Aufsteigen in der homologen Reihe; die Ammoniake nur nach der Stärke ihrer Basizität, also der Konzentration der OH-Ionen, unabhängig von der Natur des Kations, im ganzen also nach ihren Eigenschaften in wässeriger Phase. Zu diesen beiden Gruppen kommen endlich noch andere Stoffe, die ebenfalls erheblich stärker wirken, als ihrer Lipoidlöslichkeit bezw. ihrer Löslichkeit in den Zellen entspricht, wie Blausäure und arsenige Säure. Es schien von Interesse, $\mathrm{zu}$ untersuchen, ob beim Eindringen von Stoffen der genannten Gruppen in Zellen Wärmetönungen auftreten. Ist doch zu erwarten, dass bei solehen Körpern, deren Wirkungsstärke durch ihre chemische Konstitution direkt bestimmt wird, sich chemische Verbindungen bilden, wobei mehr oder weniger grosse Wärmetönungen auftreten könnten. Bei rein physikalisch wirkenden Stoffen schien dies dagegen sehr unwahrscheinlich. Sieht man von allen basischen oder sauren Substanzen ab, die wegen der unvermeidlichen Neutralisationswärme beim Vermischen mit Zellen für eine genaue Prüfung unbrauchbar sind, so ergab sich bei sämtlichen geprüften Substanzen, sowohl den indifferenten Stoffen wie den Aldehyden, dass dieselben

sation gebildeter Säuren oder fermentative Spaltungsprozesse. Für die Art und Grösse dieser Wärmetönungen scheint das Milieu weitgehend in Betracht zu kommen. Ferner erscheint es möglich, dass bei dem Aufbau der Bakterien ein Teil des Sauerstoffs in chemische Bestandteile des Bakterienleibes übergeht, wobei dann pro Einheit verbrauchten Sauerstoffs weniger Wärme als bei totaler Verbrennung des Nährmaterials entstehen würde. Auch könnte die Bildung von Stoffen mit höherer Verbrennungswärme beim Aufbau des Leibes an dem kleineren Quotienten der wachsenden Bakterien gegenüber den nicht wachsenden schuld sein. 
beim Eindringen keine messbaren Wärmetönungen zeigen, mit alleiniger Ausnahme des Formaldehyd, das schon in reversibel-atmungshemmenden Konzentrationen beim Eindringen eine beträchtliche positive Wärmetönung ergibt, während beim Thymol eine kleine innerhalb der Fehlergrenze gelegene auftrat. Die Methodik hat natürlich einen bestimmten Grenzwert der Genauigkeit, der in Kontrollmessungen festgelegt wurde: Als nicht messbare Wärmetönung gilt solche, wo beim Vermischen von $36 \mathrm{ccm}$ ganz konzentrierter Erythrocytensuspension mit 215 cem Lösung eine Temperaturveränderung von weniger als $+0,005^{\circ}$ oder $-0,01^{\circ}$ auftrat, während Temperaturveränderungen über $+0,005^{\circ}$ bis $0,015^{\circ}$ nur dann als sicher gelten können, wenn sie regelmässig beobachtet werden. In dem ersten Fall der nicht messbaren Wärmetönung sind also weniger als 1,4 cal. gebildet.

Zu dieser Gruppe gehören : Amylalkohol ${ }^{1}$ ) (1 Vol.-Proz.), Phenylurethan (gesättigte Lösung, weniger als 0,2 Proz., Chloralhydrat $(0, \dot{4}$ Proz. und 1,6 Proz.), Propylaldehyd (gesättigt, weniger als 0,48 Vol.-Proz.), Isobutyraldehyd (gesättigt, weniger als 0,36 Vol.-Proz.), Isovaleraldehyd (gesättigt, weniger als 0,15 Vol.-Proz.), Acrolein (verschiedene Konzentrationen bis zur Sättigung). Während im allgemeinen Konzentrationen gewählt wurden, die eine ganz oder teilweise reversible Atmungshemmung bewirkten (im allgemeinen letzteres, um etwas mehr Substanz verwenden zu können), erschien es daraufhin wünschenswert, zu wissen, ob sich ein Unterschied zwischen reversibler und irreversibler Hemmung geltend macht, ob insbesondere bei einer nach dem Eindringen sofort irreversiblen Hemmung, d. h. bei dem Tode der Zelle, eine Wärmetönung auftritt, die bei reversibler Hemmúng nicht stattfindet. Dieser Frage, ob der Tod von Zellen eine Wärmetönung hat, war schon in der vorherigen Arbeit nähergetreten und dieselbe verneint worden, weil beim Abtöten des Spermas mit Blausäure (erkenntlich an dem Unbeweglichwerden der Spermatozoen) keine Wärmetönung nachweisbar ist. Jedoch konnte dies deshalb angefochten werden, weil die Vergiftung mit Blausäure $\mathrm{m}$ allgemeinen reversibel und ein Wiederbeleben der Spermatozoen vielleicht nur aus technischen Gründen nicht ausführbar ist. Es wurde anstatt dessen diesmal auf den Vorschlag O. Warburg's Acrolein gewählt. Wird dasselbe in genügend hoher Konzentration

1) Alle Konzentrationen gelten vor der Vermischung. 
zu lebenden Zellen zugesetzt, so tritt eine totale Atmungshemmung ein, die bei einem wenige Minuten später stattfindenden Wegwaschen des Giftes nicht mehr zurückgeht. Bis zu diesem Wegwaschen ist aber die Temperatur beobachtet. Da in dieser Zeit das "Irreversibelwerden der Hemmung", also der Tod, stattgefunden hat, so hätte eine grössere hierbei auftretende Wärmetönung der Feststellung nicht entgehen können. Indes bestätigt der Versuch das frühere Resultat: Auch bei Zusatz von Acrolein in tödlicher Konzentration zu lebenden Zellen tritt keine messbare Wärmetönung a uf. Wenn also in der Sprache vieler Physiologen beim Tode "lebende Moleküle zerfallen", so findet dabei kein mit obiger Methode messbarer Energieverlust statt. Dieser Feststellung, dass ein Energiepotential des Lebensprozesses nicht erkennbar ist, könnte als Einwand die relativ grosse Unempfindlichkeit des Messungsverfahrens entgegengehalten werden, da ja die "Bedeutung" der fraglichen energetischen Grösse nichts mit ihrem absoluten Maasse zu tun zu haben brauchte. Indes dürfte sich durch die Versuche jedenfalls eine Idee ausschliessen lassen, die kürzlich von $\mathrm{Zuntz}$ im Anschlusse an den Befund des so sehr kleinen kalorischen Quotienten beim Seeigelei erörtert wurde ${ }^{1}$ ). Zuntz hält es im Anschluss an die Li e bi g-Pfl ü g er'sche Lehre für möglich, dass beim Oxydationsprozesse im Seeigelei der aus dem Sauerstoffverbrauch berechnete Verlust an chemischer Energie der Eisubstanz deshalb nicht vollständig als Wärme frei wird, weil ein Teil derselben zur Bildung belebten Eiweisses aus unbelebtem verwendet werden könnte. Diese im lebenden Eiweiss aufgestapelte Energie müsste dann aber im Tode als Wärme frei werden. Berechnet man nun aus den früher angegebenen Daten, ein wie grosser Bruchteil der chemischen Energie innerhalb der gemessenen 14 Stunden bei der Furchung des Seeigeleis in das Eiweiss investiert sein müsste, so findet man, dass pro $140 \mathrm{mg} \mathrm{N}$ schon in dieser Zeit etwa 30-35 cal. nicht ersehienen sind. Wenn man nun auch nicht die Annahme machen will, dass das Eiweiss in lebenden Zellen in seiner "Belebtheit" ganz gleichwertig ist, so wird man doch jedenfalls den Grad derselben nach der Atmungsgrösse abschätzen dürfen. Nun atmet allerdings $1 \mathrm{mg} N$ der Gänse-Erythrocyten in der Zeiteinheit nur etwa $1 / 20$ von $1 \mathrm{mg} \mathrm{N}$ der sich furchenden Seeigeleier im Mittel. Aber auch dann müssten noch

1) Handb. d. Biochemie von Oppenheimer Bd. 4 (1) S. 831. 
beim Tode von $140 \mathrm{mg} \mathrm{N}$ von Vogel-Erythrocyten 1,5 cal. auftreten. $1 \mathrm{ccm}$ der hier benutzten Erythrocyten-Suspension enthält ungefähr $56 \mathrm{mg} \mathrm{N}, 36 \mathrm{ccm}$ demnach $2016 \mathrm{mg} \mathrm{N}$. Beim Tode dieser Zellenmenge hätten also 23 cal. bzw. $0,085^{\circ}$ Temperaturerhöhung auftreten müssen.

Die Methode ist im wesentlichen dieselbe, wie sie bei den Versuchen über das Eindringen der Kohlensäure in Rinder-Erythrocyten benutzt wurde. Doch fanden sämtliche Versuche im Thermostaten von $29^{\circ}$ statt. Die Erythrocytensuspension befand sich stets im inneren Glasrohr. Vor dem Herausstossen des Glasstöpsels zum Vermischen der Aussen- und Innenflüssigkeit wurde in allen Fällen mindestens 2 Stunden gewartet, was sich in Vorversuchen für einen totalen Temperaturausgleich als nötig erwies. Diese Zeit ist deshalb noch länger wie bei den oben beschriebenen Versuchen, weil infolge der Atmung der Erythrocyten eine sich nur sehr langsam und nicht immer total ausgieichende Temperaturdifferenz vorhanden ist. Aus diesem Grunde ist auch die Genauigkeitsgrenze nach unten weiter angegeben als nach oben, weil oft infolge des durch die Atmung bedingten Temperaturgefälles im Gefäss die Innenlösung 0,002 bis $0,005^{0}$ wärmer ist als die Aussenlösung. Andererseits können durch kräftige Rührung, wie sie für ein sofortiges Vermischen nötig ist, Temperaturanstiege um $0,003^{\circ}$ stattfinden. Den Grad der beabsichtigten Atmungshemmung konnte man nicht aus der wirksamen Konzentration der Stoffe entnehmen, weil sich die Mehrzahl der Substanzen stark in der Zelle anhäuft und sich dadurch die Konzentration beim Vermischen mit Blut stark vermindert. Durch die neuen Verteilungversuche von Warburg (zum Teil nach nicht veröffentlichten Versuchen) waren hierfür Anhaltspunkte gegeben. Im übrigen kam es bei Stoffen ohne Wärmetönung nicht darauf an, etwas zu viel zu nehmen. Im allgemeinen wurde etwa die doppelte Menge genommen, als sie sich für eine Atmungshemmung um $50 \%$ berechnen liess.

\section{Versuche.}

Thymol: drei Versuche mit $0,015 \%$, zweimal um $+0,010^{\circ}$, einmal unter $+0,005^{\circ}$; zwei Versuche mit $0,06 \%$, einmal $+0,012^{\circ}$, einmal $0,00^{\circ}$.

Formaldehyd (nach Versuchen von Warburg löst sich Formaldehyd etwa zehn bis zwölfmal so stark in Gänse-Erythrocyten wie in der umgebenden Lösung).

1. 0,012 Vol.-Proz. gibt $+0,01$ bis $0,02^{\circ}$ (ungenauer Versuch).

2. 0,024 Vol..Proz. gibt $+0,030^{\circ}$.

3. 0,024 Vol.-Proz. gibt $+0,035-0,040^{\circ}$. Die Atmungshemmung ist teilweise reversibel.

4. Versuch in anderer Anordnung. Zu $200 \mathrm{~cm}$ Blutkörperchensuspension, deren Sauerstoff durch Wasserstoffdurchleiten grösstenteils entfernt ist, $2 \mathrm{ccm}$ $40 \%$ iger Formaldehydlösung zugesetzt. Temperaturanstieg gemessen $+0,11^{\circ}$, + Korr. ungefähr $0,03^{\circ}$, zusammen ca. $0,14^{\circ}$.

5. Formaldehyd $0,05 \%$ za Gänseserum gesetzt, desgleichen $0,1 \%$; es ergibt sich in beiden Fällen ein Temperaturanstieg um $+0,005^{\circ}$. Möglicherweise 
184 O. Meyerh of: Über Wärmetönungen chem. Prozesse in Iebənden Zellen.

findet also eine geringe Wärmebildung mit Serum statt. Da beim Einfliessen von Formol in Kochsalzlösung keine Temperaturveränderung erfolgt, wäre sie auf eine chemische Einwirkung auf das Eiweiss zurückzuführen.

Acrolein. 1) $\mathrm{Zu} 150 \mathrm{ccm}$ Enten-Erythrocyten (in D IV) in Phenylurethan wird $0,02 \mathrm{ccm} 33 \%$ ige Lösung von Acrolein gefügt. Es findet keine Temperaturänderung statt.

2) $\mathrm{Zu} 60 \mathrm{ccm}$ Erythrocytensuspension, auf $240 \mathrm{ccm}$ verdünnt, wird nacheinander $0,04,0,05$ und $0,06 \mathrm{ccm} 33 \%$ iger Acroleinlösung gefügt. Die Wärmebildung (Atmung) wird durch jeden weiteren Zusatz stärker herabgedrückt, aber selbst durch die Gesamtmenge $0,15 \mathrm{ccm}$ nicht aufgehoben. Beim Einspritzen keine Temperaturveränderungen. (Kontrollversuche zeigen, dass in gleichen Mengenverhältnissen die Sauerstoffzehrung ähnlich beeinflusst wird, wie dies an den Wärmeversuchen gefunden ist).

3) Gewöhnliche Anordnung: $36 \mathrm{ccm}$ konzentrierte Gänse-Erythrocyten, vermischt mit $215 \mathrm{ccm}$ gesättigter Lösung (annähernd $0,03 \%$ ) von Acrolein. Temperaturveränderung beim Durchstossen $0,000^{\circ}$, ebenso bei 10 Minuten Warten. 12 Minuten nachher ist das Acrolein aus einer Blutkörperchenprobe weggewaschen, während eine andere in der Acroleinlösung auf das entsprechende Volumen gebracht wird. Beide werden in Atmungsgläschen von $6,3 \mathrm{ccm}$ gefüllt und 4 Stunden im Thermostaten bei $29^{\circ}$ gelassen. Die Sauerstoffzehrung beträgt für das ungewaschene Blut in dieser Zeit $v=31, m=6,2, t=17^{\circ}, p=11 \mathrm{~mm}$. Für das gewaschene $v, m, t$, desgl. $p=6 \mathrm{~mm}$, während sich aus einem vorher gemachten Kontrollversuch für die gleiche Menge der gleichen Suspension nicht behandelten Blutes in derselben Zeit $p=215 \mathrm{~mm}$ ergibt. Daraus folgt, dass die Erythrocyten getötet sind.

4) Eine Wiederholung des Versuches ergibt eine Temperaturveränderung von $+0,001^{\circ}$ and ebenfalls eine total irreversible Atmungshemmung.

Zum Schlusse möchte ich Herrn Dr. Otto Warburg für die vielfache freundliche Beratung herzlichen Dank sagen. 